\title{
The Usefulness of Platelet-Rich Plasma (PRP) for the Treatment of Vitiligo: State of the Art and Review
}

This article was published in the following Dove Press journal:

Drug Design, Development and Therapy

\section{SR Mercuri ${ }^{1}$ \\ L Vollono $\left.^{2}\right)^{2}$ \\ G Paolino $\mathbb{D}^{1,3}$}

'Unit of Dermatology, IRCCS, Ospedale San Raffaele, Milan, Italy; ${ }^{2}$ Dermatology Unit, Department of "Medicina dei Sistemi", University of Rome Tor Vergata, Rome, Italy; ${ }^{3}$ Dermatologic Clinic, La Sapienza University of Rome, Rome, Italy
Correspondence: L Vollono

Tor Vergata University, via Cracovia 50, Rome 00133, Italy

Tel +390620902743

Fax +390620902742

Email laura.vollono@gmail.com
Introduction: Vitiligo is an acquired, idiopathic disorder clinically characterized by amelanotic lesions on the skin which cause significant impairment of patients' quality of life. A variety of treatments have been proposed, with inconsistent results. In the last decades, the use of platelet-rich plasma (PRP) is receiving increasing interest as a potential effective technique in the treatment of several dermatological diseases, including vitiligo.

Objective: We conducted a review with the aim to identify studies that documented the use of PRP for vitiligo.

Materials and Methods: Electronic databases of MEDLINE, EMBASE and Cochrane Central Register of Controlled Trials (CENTRAL) from inception to November 2019 have been searched using different combinations of the following terms: "platelet-rich plasma", "platelet gel", "platelet-rich fibrin", "PRP" and "vitiligo".

Results: We identified 6 clinical studies consistent with our research, with a total of 253 patients, listing and discussing the obtained results. In all reports, all treated patients showed a stable vitiligo, and a significantly higher improvement in the PRP groups was always observed compared to control groups. Regarding the side effects, PRP in vitiligo patients is useful and without important side effects.

Conclusion: PRP is a promising treatment for stable vitiligo lesions in different body sites. The possible use of PRP in combination with traditional therapeutic options and the standardization of processing protocols represents a very fertile field for future research. Larger clinical trials with longer time of observation would provide solid evidence regarding the effectiveness of PRP for the treatment of vitiligo.

Keywords: vitiligo, skin, platlet rich plasma, PRP, treatment

\section{Introduction}

Vitiligo is an acquired, idiopathic disorder clinically characterized by amelanotic lesions on the skin, due to destruction and subsequent absence of melanocytes. ${ }^{1}$ The prevalence of vitiligo is between $0.4 \%$ and $4 \%$, with a positive familiar history in about $30 \%$ of cases. ${ }^{2,3}$ Although vitiligo is not a life-threatening disorder, about $1 / 3$ of patients with vitiligo suffers from psychological disorders, with significant impairment of quality of life. ${ }^{2}$ Accordingly, several efforts should be made in order to identify appropriate and effective treatments.

The cause of vitiligo is not yet fully understood. ${ }^{3}$ Several hypotheses regarding the pathogenesis of the disease exist, although none has been confirmed as the main one. These main hypotheses include autoimmune hypothesis, neural hypothesis, self-destruct 
hypothesis and biochemical hypothesis ${ }^{3}$ Based on each of these hypotheses, various treatments have been developed in the last decades, in order to increase the therapeutic success, reducing relapses and improving patients' quality of life. ${ }^{3}$ Corticosteroid, calcineurin inhibitors and 308 nm-excimer laser play a role in active and localized disease, inducing immunosuppression; photo-chemotherapy, UVB phototherapy and narrowband UVB phototherapy are also other valid treatments for generalized vitiligo. ${ }^{4}$ While surgery techniques are mainly based on autologous transplanting of melanocytes. ${ }^{1,5}$ However, despite the presence of several therapeutic options, all these therapeutic modalities show limited responses. ${ }^{3}$

\section{Platelet-Rich Plasma (PRP)}

PRP is an increased concentration of autologous platelets suspended in a small amount of plasma, after centrifugation. ${ }^{6,7}$ The production of PRP starts with the collection of 10-60 mL venous blood, the same day of the treatment. Before the centrifugation, anticoagulants are usually added to prevent coagulation and premature secretion of alpha granules. ${ }^{7}$ According to their cell content and fibrin architecture, up to date, the are four different types of PRP preparation: I) Pure Platelet-Rich Plasma, a leucocyte-poor PRP with low-density fibrin network after activation; II) Leucocyte-PRP, a leucocyte-rich PRP with low-density fibrin network; III) Pure Platelet-Rich Fibrin, a leucocyte-poor PRP with high-density fibrin network; IV) Leucocyte and Platelet-Rich Fibrin, a leucocyte-rich PRP with high-density fibrin network. ${ }^{8,9}$

Through the secretion of platelet's alpha granules, PRP increases the release of growth factors, adhesion molecules and chemokines, which interacting with the local environment, promotes cell differentiation, proliferation and regeneration. ${ }^{7}$ (Figure 1) The main platelet growth factors secreted are: platelet-derived growth factor (PDGF) a-b, transforming growth factor (TGF), alpha-beta, vascular endothelial growth factor (VEGF), epidermal growth factor (EGF), fibroblast growth factor (FGF), connective tissue growth factor (CTGF) and insulin-like growth factor-1 (IGF-1). ${ }^{8}$ Usually, the active secretion of these growth factors by platelets begins within 10 min after activation. ${ }^{8,10}$

Moreover, PRP promotes the release of inflammatory mediators and modulators. Indeed, platelets may release numerous anti-inflammatory cytokines, such as IL-1 receptor antagonist (IL-1 ra), soluble tumor necrosis factor (TNF) receptor (sTNF-R) I, IL-4, IL-10, IL-13 and interferon $\gamma .^{11}$

To date, no standard protocols regarding PRP preparation exist. Published studies report variations in processing, such as the number of centrifugations or compounds

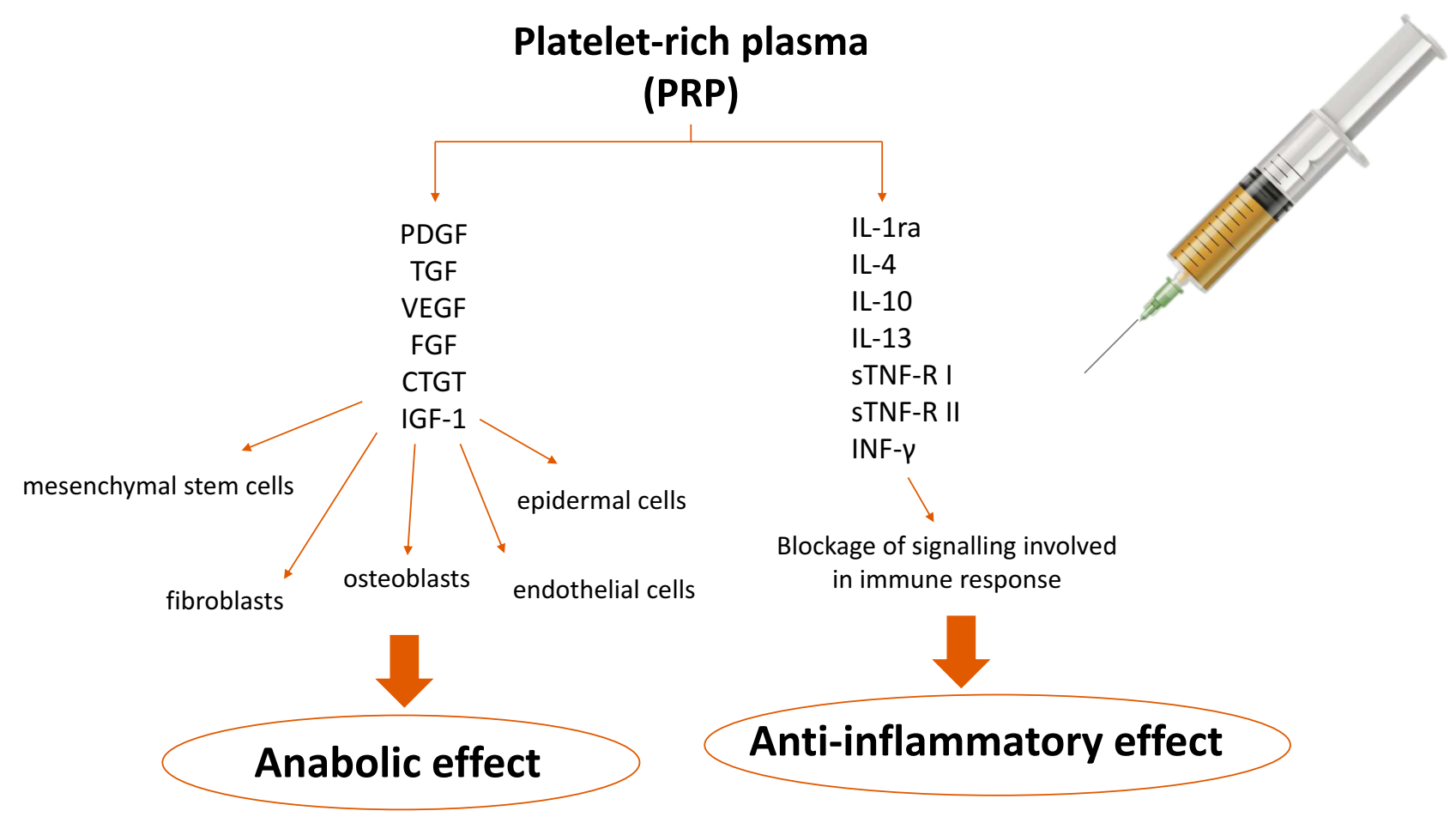

Figure I Effects of PRP on growth factors, cytokines, chemokines, inflammatory mediators and modulators. 
added (Table 1), which make it difficult to compare results from different clinical studies. In this regard, randomized, comparative clinical trials are needed in order to establish the recommended processing protocol.

The aim of this review is to evaluate the usefulness of Platelet-Rich Plasma (PRP) for the treatment of vitiligo, since the interest in this new treatment is growing among the therapeutic options for vitiligo.

\section{Materials and Methods}

We conducted a review to identify studies that documented the use of PRP for vitiligo. All type of study, in English language, was considered eligible for this review, including case reports and case series. The main search was conducted in the electronic databases of MEDLINE, EMBASE and Cochrane Central Register of Controlled Trials (CENTRAL) from inception to November 2019 using different combinations of the following terms: "platelet-rich plasma", "platelet gel", "platelet-rich fibrin", "PRP" and "vitiligo". Additionally, we concluded the manual search by reviewing all the relevant citations within the identified articles.

\section{Results}

We herewith summarize the obtained results, taking into account study design, number of enrolled patients, vitiligo characteristics, type of intervention, PRP preparation process, outcome evaluation score, time of follow-up, histopathological evaluation and reported side effects (Table 1).

Specifically, six clinical studies regarding the use of PRP in vitiligo have been identified, with a total of 253 patients. ${ }^{1,12-16}$ The mean time of follow-up of treated patients was 6 months (ranging between 3 and 12 months). ${ }^{1,12-16}$ In all reports, ${ }^{1,12-16}$ all treated patients showed a stable vitiligo, and a significantly higher improvement in the PRP groups was always observed compared to control groups, regardless of the combined treatment associated with PRP. ${ }^{1,12-16}$ Regarding the side effects, PRP in vitiligo patients is safe, without important and specific side effects. ${ }^{1,12-16}$ Pain at the injection site was the main side effect, although it can be avoided applying 45-60 minutes before the injection of an anesthetic cream. ${ }^{16}$ Besides, in order to avoid local superinfection, topical antibiotics can be used 3 days after injection. ${ }^{16}$ Ecchymosis in the site of injection may occur. ${ }^{14}$ Ejjiyar et al reported the onset of Koebner's phenomenon in a female patient phototype IV with the onset of facial non-segmental vitiligo after the third injection of PRP, for aesthetic purposes. ${ }^{17}$

\section{Discussion}

Ibrahim et al evaluated the effect of PRP on the outcome of short-term NB-UVB therapy for patients with stable vitiligo. ${ }^{12}$ The authors enrolled 60 patients (26M:34F; mean age of 28), with symmetrical stable lesions. For each patient, the left side of the body was treated with NB-UVB alone, while the right side was treated with NB-UVB therapy in addition to intradermal injection of PRP. ${ }^{12}$ In order to evaluate the response, the authors performed a score, as follows: $>75 \%$ to $100 \%$ (excellent); between $50 \%$ and $75 \%$ (good); between $25 \%$ and $50 \%$ (moderate) and $<25 \%$ (mild). ${ }^{12}$ After 3 months, two independent dermatologists evaluated the patients, reporting an excellent response in $55 \%$ of the lesions treated with PRP $(p<0.01) .{ }^{12}$ Besides after 7 months of follow-up in the treated lesions, there was no relapse, contrariwise to the control side. ${ }^{12}$ Finally, histological analysis confirmed the increase in the number of melanocytes in treated lesions. ${ }^{12}$

Abdelghani et al evaluated a combined treatment with fractional carbon dioxide laser, autologous PRP and narrowband ultraviolet $\mathrm{B}$ in different body sites, in 80 patients with vitiligo for 12 months. ${ }^{13}$ Specifically, the patients were randomly categorized in four different groups: fractional $\mathrm{CO} 2$ laser group, PRP group, combined fractional $\mathrm{CO} 2$ laser + PRP and combined fractional $\mathrm{CO} 2$ laser + NB-UVB. ${ }^{13}$ Patients included in combined fractional $\mathrm{CO} 2$ laser + PRP reached the best results regarding repigmentation; specifically $60 \%$ of the patients developed a repigmentation $>50 \%$ and $40 \%$ of patients developed a repigmentation $>75 \% .{ }^{13}$

Kadry et al evaluated PRP versus fractional $\mathrm{CO} 2$ laser and combined fractional $\mathrm{CO} 2$ laser + PRP, followed by sun exposure for the treatment of stable no segmental vitiligo in 30 patients (22F:8M; mean age of 32 years). ${ }^{14}$ Intrapatient lesions were randomly divided into 4 different groups: PRP group, combined fractional $\mathrm{CO} 2$ laser + PRP, fractional $\mathrm{CO} 2$ laser group and control group. ${ }^{14}$ Subsequently, all patients have been evaluated according to vitiligo analysis by computer-assisted grid (VACAG), mean improvement score by physician (MISP) and visual analogic scale (VAS). ${ }^{14}$ Patients included in the combined fractional CO2 laser + PRP group and patients included in the PRP group showed a significant improvement compared to the other treatments $(\mathrm{p}<0.001)^{14}$ Also histology revealed a prominent repigmentation, confirmed also by HMB $45 .{ }^{14}$ 


\begin{tabular}{|c|c|c|c|}
\hline 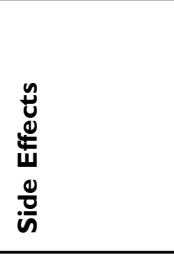 & 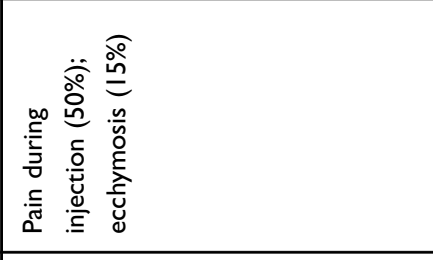 & 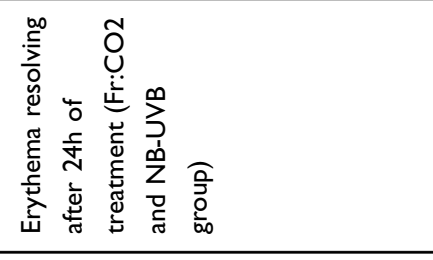 & 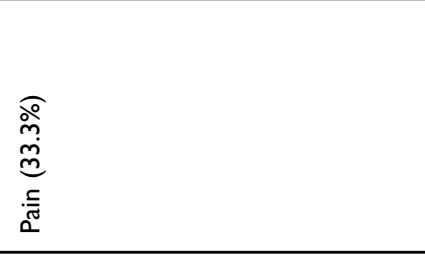 \\
\hline 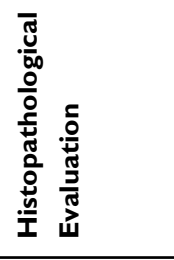 & 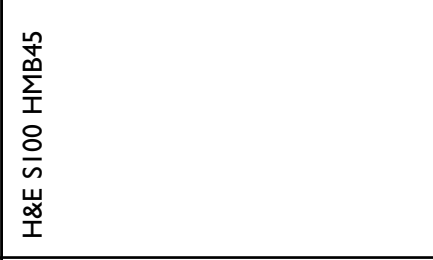 & $\frac{\infty}{z}$ & $\begin{array}{l}\text { 号 } \\
\text { 岕 } \\
\text { I }\end{array}$ \\
\hline 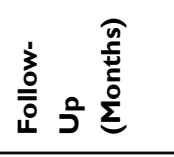 & $\simeq$ & $\mathrm{m}$ & \\
\hline $\begin{array}{l}\frac{n}{\underline{\underline{z}}} \\
\stackrel{\vec{z}}{x}\end{array}$ &  & 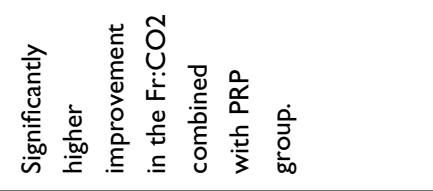 & 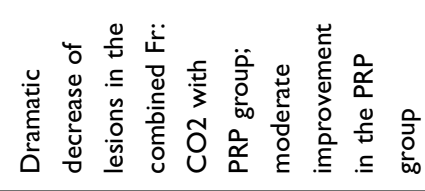 \\
\hline 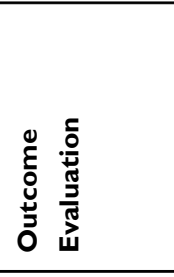 & 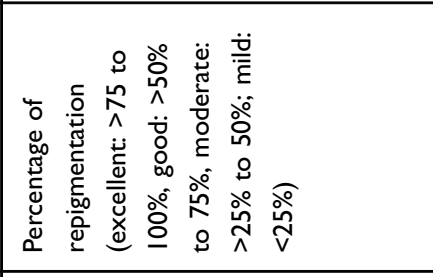 & 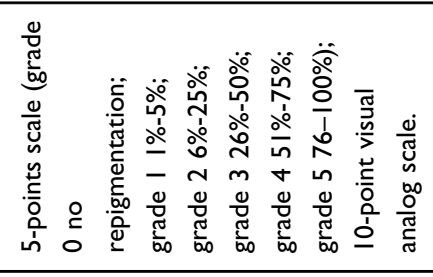 & 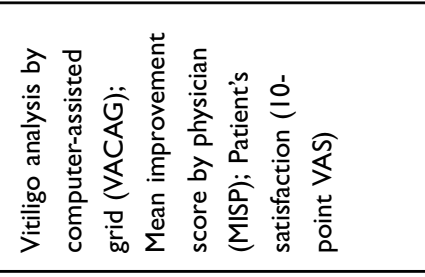 \\
\hline 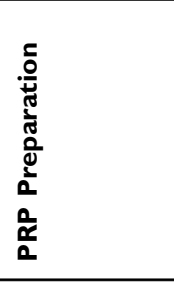 & 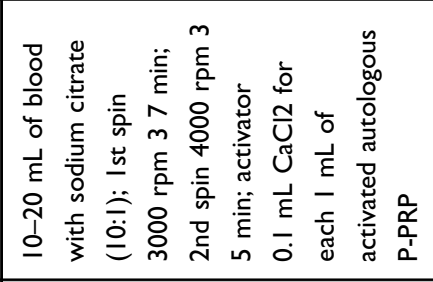 & 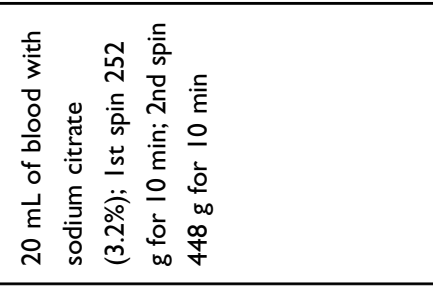 & 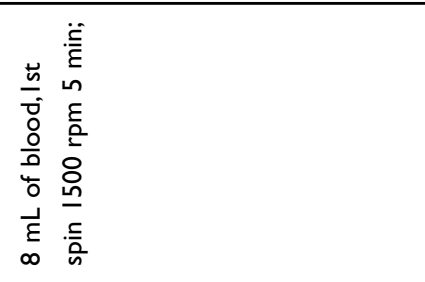 \\
\hline 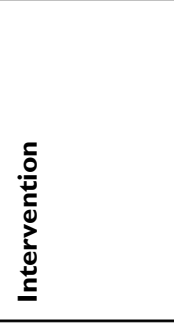 & 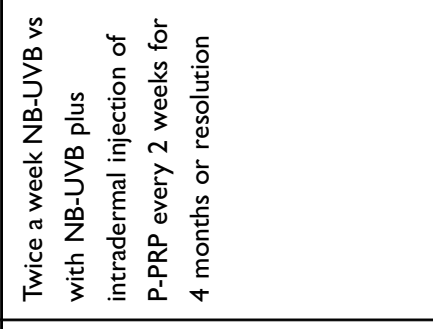 & 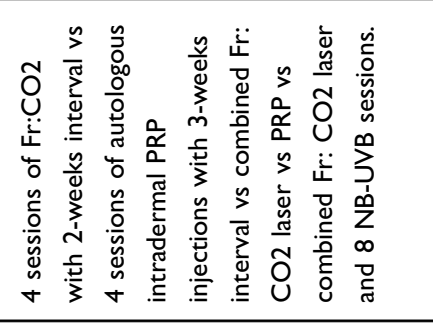 & 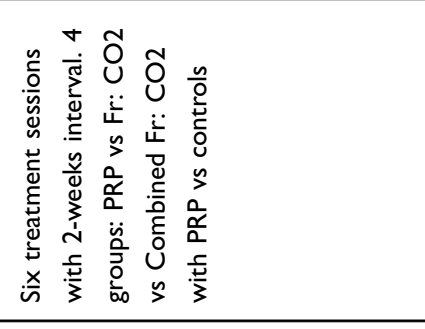 \\
\hline 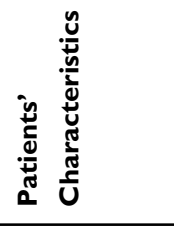 & 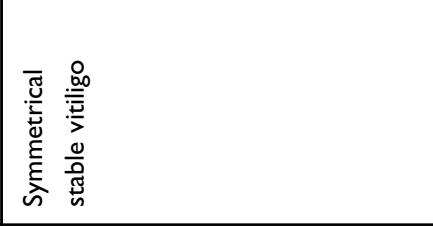 &  & 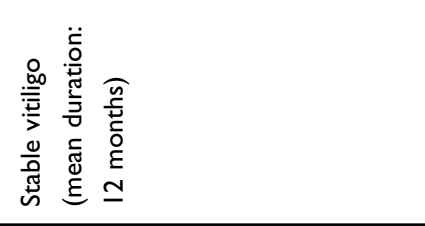 \\
\hline 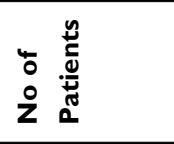 & 8 & $\infty$ & $\stackrel{m}{n}$ \\
\hline 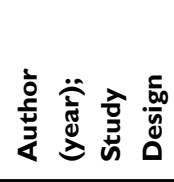 & 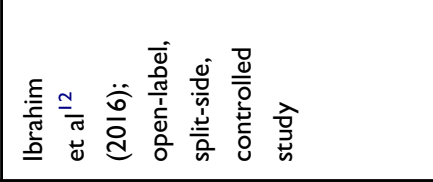 & 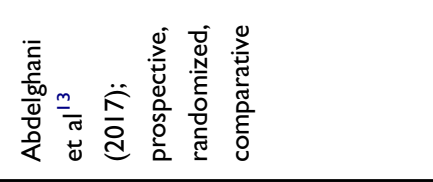 & 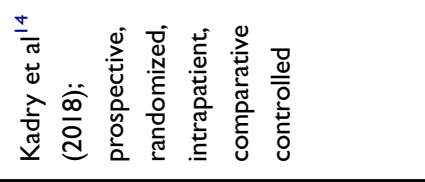 \\
\hline
\end{tabular}




\begin{tabular}{|c|c|c|}
\hline 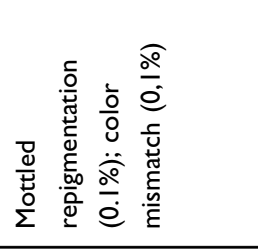 & 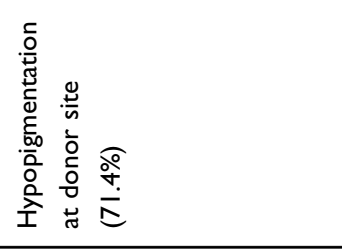 & 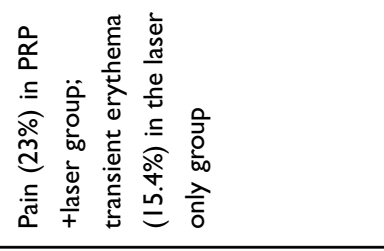 \\
\hline 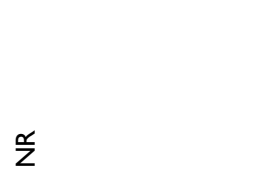 & $\stackrel{\alpha}{Z}$ & 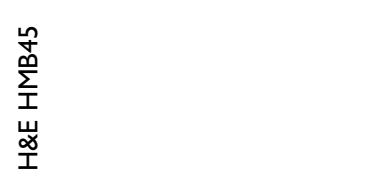 \\
\hline 0 & 0 & $m$ \\
\hline 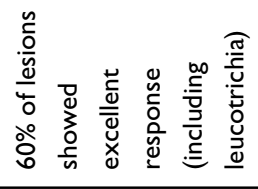 & 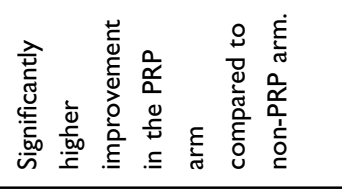 & 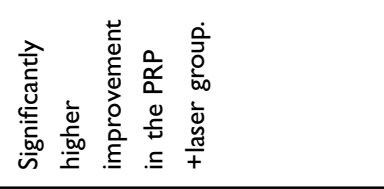 \\
\hline 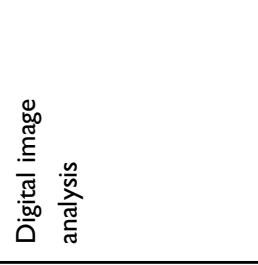 & 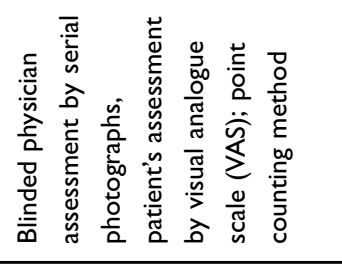 & 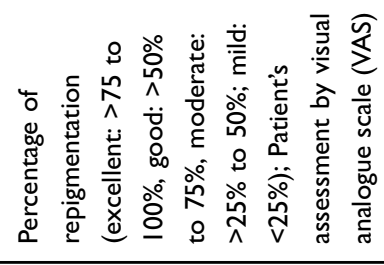 \\
\hline 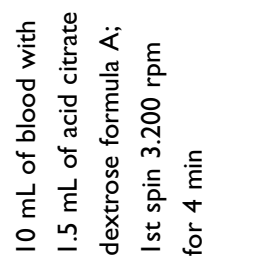 & 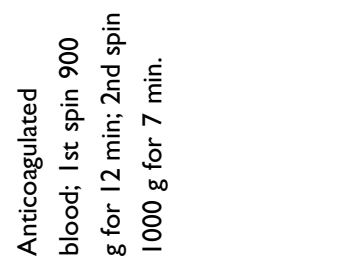 & 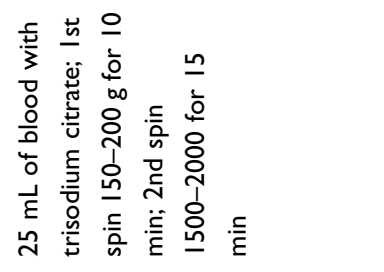 \\
\hline 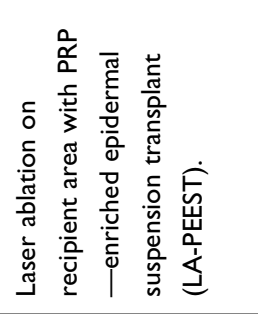 &  & 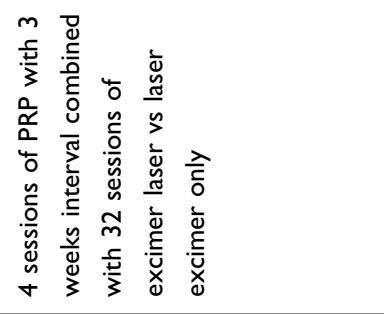 \\
\hline 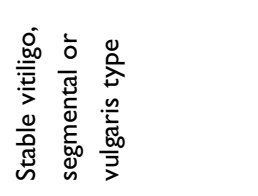 & 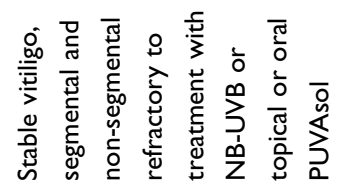 & 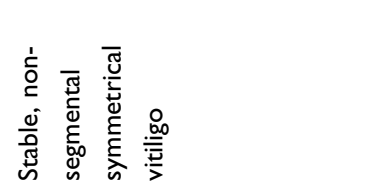 \\
\hline 으 & $\bar{\sim}$ & กี \\
\hline 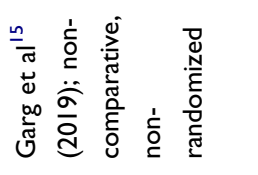 & 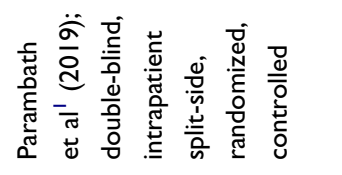 & 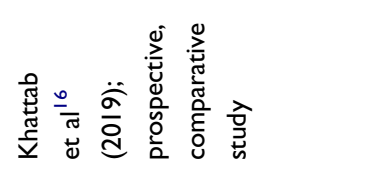 \\
\hline
\end{tabular}


Garg et al evaluated the extent of repigmentation with non-cultured trypsinized fragmented epidermal suspension using PRP and pixel erbium yttrium aluminium garnet laser for ablation of recipient areas in 10 patients with stable vitiligo. ${ }^{15}$ The authors found an excellent response in $60 \%$ of patients; the repigmentation was higher in the legs, knees, face and neck, as well as an improvement of leucotrichia was found. ${ }^{15}$

In another study, Parambath et al compared the extent of repigmentation achieved by transplantation of nocultured epidermal cell suspension (NCES) suspended in PRP and NCES suspended in phosphate buffered saline (PBS), in 21 patients (13F:8M; mean age of 23.1) with stable vitiligo, characterized by at least two lesions of comparable size. ${ }^{1}$ Subsequently, after 1 week, the patients performed heliotherapy for 15 minutes, daily. ${ }^{1}$ After 6 months of follow-up, the repigmentation in treated areas with PRP was $75.6 \%$, while it was $65 \%$ in non-treated areas with PRP $(p=0.0036)$.

Recently, Khattab et al included in a study of 52 patients (8M:44F), with stable, no segmental and symmetrical vitiligo, randomized in two different groups in order to evaluate the efficacy of the treatment with combined excimer laser and PRP. ${ }^{16}$ Specifically, in the first group, the patients were treated with intradermal PRP injection and excimer laser (PRP after excimer laser every 3 weeks for a total of six sessions of PRP), while in the second group, the patients were treated with only excimer laser. A better response was reached in the first group, also according to VAS score. ${ }^{16}$

\section{Conclusions}

PRP is a well-tolerated agent, recently receiving increasing attention by the medical community for its potential use in several dermatological conditions, including vitiligo. Literature confirms PRP as a safe and promising treatment for stable vitiligo lesions in different body sites, above all when PRP is combined with other physical procedures, such as fractional carbon dioxide laser. Four-six sessions, with 2-3-week interval are needed in order to obtain clinically significant results. However, the lack of consensus regarding preparation methods, it makes difficult to compare results from different clinical studies. Larger clinical trials with longer time of observation and the standardization of processing protocols represent a very fertile field for future research about the effectiveness of PRP for the treatment of vitiligo.

\section{Acknowledgments}

The authors wish to thank Mrs Giada Moffa and Mrs Caterina Prezioso for their significant contribution to this paper.

\section{Disclosure}

The authors report no conflicts of interest in this work.

\section{References}

1. Parambath N, Sharma VK, Parihar AS, et al. Use of platelet-rich plasma to suspend noncultured epidermal cell suspension improves repigmentation after autologous transplantation in stable vitiligo: a double-blind randomized controlled trial. Int $J$ Dermatol. 2019;58:472-476. doi:10.1111/ijd.14286

2. Bidaki R, Haghighi FS. Recurrent suicide attempt in two sisters with non-segmental vitiligo and obesity: a rare case report. Pigment Disord. 2016;3:235. doi:10.4172/2376-0427.1000235

3. Forschner T, Buchholtz S, Stockfleth E. Current state of vitiligo therapy-evidence-based analysis of the literature. $J$ Dtsch Dermatol Ges. 2007;5:467-475. doi:10.1111/j.1610-0387.2007.06280.x

4. Bae JM, Jung HM, Hong BY, et al. Phototherapy for vitiligo: a systematic review and meta-analysis. JAMA Dermatol. 2017;153:666-674. doi:10.1001/jamadermatol.2017.0002

5. Zokaei S, Farhud DD, Keykhaei M, et al. Cultured epidermal melanocyte transplantation in vitiligo: a review article. Iran J Public Health. 2019;48:388-399.

6. Wang HL, Avila G. Platelet rich plasma: myth or reality? Eur J Dent. 2007;1:192-194. doi:10.1055/s-0039-1698338

7. Hesseler MJ, Shyam N. Platelet-rich plasma and its utility in medical dermatology: a systematic review. $J$ Am Acad Dermatol. 2019;81:834-846. doi:10.1016/j.jaad.2019.04.037

8. Dhurat R, Sukesh M. Principles and methods of preparation of platelet-rich plasma: a review and author's perspective. J Cutan Aesthet Surg. 2014;7:189-197. doi:10.4103/0974-2077.150734

9. Dohan Ehrenfest DM, Rasmusson L, Albrektsson T. Classification of platelet concentrates: from pure platelet-rich plasma (P-PRP) to leucocyte- and platelet-rich fibrin (L-PRF). Trends Biotechnol. 2009;27:158-167. doi:10.1016/j.tibtech.2008.11.009

10. Marx RE. Platelet-rich plasma (PRP): what is PRP and what is not PRP? Implant Dent. 2001;10:225-228. doi:10.1097/00008505200110000-00002

11. Woodell-May J, Matuska A, Oyster M, et al. Autologous protein solution inhibits MMP-13 production by IL-1beta and TNFalpha-stimulated human articular chondrocytes. J Orthop Res. 2011;16:1320-1326. doi:10.1002/jor.21384

12. Ibrahim ZA, El-Ashmawy AA, El-Tatawy RA, et al. The effect of platelet-rich plasma on the outcome of short-term narrowband-ultraviolet B phototherapy in the treatment of vitiligo: a pilot study. J Cosmet Dermatol. 2016;15:108-116. doi:10.1111/ jocd. 12194

13. Abdelghani R, Ahmed NA, Darwish HM. Combined treatment with fractional carbon dioxide laser, autologous platelet-rich plasma, and narrow band ultraviolet $\mathrm{B}$ for vitiligo in different body sites: a prospective, randomized comparative trial. J Cosmet Dermatol. 2017;17:365-372. doi:10.1111/jocd.12397

14. Kadry M, Tawfik A, Abdallah N, et al. Platelet-rich plasma versus combined fractional carbon dioxide laser with platelet-rich plasma in the treatment of vitiligo: a comparative study. Clin Cosmet Investig Dermatol. 2018;11:551-559. doi:10.2147/CCID.S178817

15. Garg S, Dosapaty N, Arora AK. Laser ablation of the recipient area with platelet-rich plasma-enriched epidermal suspension transplant in vitiligo surgery: a pilot study. Dermatol Surg. 2019;45:83-89. doi:10.1097/DSS.0000000000001641 
16. Khattab FM, Abdelbary E, Fawzi M. Evaluation of combined excimer laser and platelet-rich plasma for the treatment of nonsegmental vitiligo: a prospective comparative study. J Cosmet Dermatol. 2019. Epub ahead of print.
17. Ejjiyar M, Sahibi M, El Gueouatri M, et al. Vitiligo et phénomène de Koebner suite à des injections de plasma riche en plaquettes [Vitiligo and Koebner phenomenon following platelet-rich plasma injections]. Pan Afr Med J. 2019;32:58. doi:10.11604/pamj.2019.32.58.16779

\section{Publish your work in this journal}

Drug Design, Development and Therapy is an international, peerreviewed open-access journal that spans the spectrum of drug design and development through to clinical applications. Clinical outcomes, patient safety, and programs for the development and effective, safe, and sustained use of medicines are a feature of the journal, which has also been accepted for indexing on PubMed Central. The manuscript management system is completely online and includes a very quick and fair peer-review system, which is all easy to use. Visit http://www. dovepress.com/testimonials.php to read real quotes from published authors.

Submit your manuscript here: https://www.dovepress.com/drug-design-development-and-therapy-journal 